\title{
Pauli Blocking of Collisions in a Quantum Degenerate Atomic Fermi Gas
}

\author{
B. DeMarco, S. B. Papp, and D. S. Jint \\ $J I L A$, \\ National Institute of Standards and Technology and University of Colorado \\ and \\ Physics Department, University of Colorado, Boulder, CO 80309-0440
}

(October 25, 2018)

\begin{abstract}
We have produced an interacting quantum degenerate Fermi gas of atoms composed of two spin-states of magnetically trapped ${ }^{40} \mathrm{~K}$. The relative Fermi energies are adjusted by controlling the population in each spin-state. Measurements of the thermodynamics reveal the resulting imbalance in the mean energy per particle between the two species, which is as large as a factor of 1.4 at our lowest temperature. This imbalance of energy comes from a suppression of collisions between atoms in the gas due to the Pauli exclusion principle. Through measurements of the thermal relaxation rate we have directly observed this Pauli blocking as a factor of two reduction in the effective collision cross-section in the quantum degenerate regime.
\end{abstract}

PACS numbers: 03.75.-b, 05.30.Fk, 05.70.Ln

Typeset using REVTEX 
The experimental realization of a quantum degenerate Fermi gas of atoms [1] introduces a novel tool for the study of quantum phenomena. The initial work, which extended the evaporative cooling and magnetic trapping techniques used for atomic Bose-Einstein condensation, produced a nearly ideal Fermi gas. Although the lack of interparticle interactions makes theoretical interpretation straightforward and may facilitate precision measurements [2], a richer spectrum of phenomena can arise in a dilute, interacting system. Predicted phenomena for an interacting atomic Fermi gas include zero sound [3], suppression of elastic and inelastic collisions [4,5], component separation [6], and the prospect of a paired state at low temperature [6,7], similar to Bardeen-Cooper-Schrieffer (BCS) superconductivity. Furthermore, unique opportunities exist for investigation of interaction physics in the atomic system via techniques that have been established for fundamental control over atomic interactions [8]. In this Letter we present measurements of the equilibrium thermodynamic properties and collisional dynamics of an interacting Fermi gas composed of ${ }^{40} \mathrm{~K}$ atoms in two spin-states.

Producing a two-component Fermi gas, where both species are quantum degenerate, builds on the earlier experimental procedure [1]. Fermionic atoms are magnetically trapped in two spin-states (magnetic sub-levels $m_{f}=9 / 2$ and $m_{f}=7 / 2$ in the ${ }^{40} \mathrm{~K} f=9 / 2$ hyperfine ground state) to allow the s-wave collisions necessary for rethermalization during evaporative cooling [9]. At low temperature, this mixture of spin-states is stable against spin relaxation and $m_{f}=9 / 2$ and $m_{f}=7 / 2$ atoms can only collide with each other 4 . Previously, the last stage of evaporation slowly removed the $m_{f}=7 / 2$ atoms to sympathetically cool the remaining $m_{f}=9 / 2$ atoms into the quantum degenerate regime. This last stage was eliminated by improving the simultaneous evaporation of the two spin-state mixture so that an equal mixture can now be cooled to $T / T_{F} \approx 0.25$, where $T$ is the temperature and $T_{F}$ the Fermi temperature. Improvements include increased stability of the magnetic trapping field, elimination of atoms in spin-states other than $m_{f}=9 / 2$ and $m_{f}=7 / 2$, and increased microwave power for the evaporative knife. A technique has also been implemented to simultaneously image both components in order to extract information about the interacting gas. During 
the $10 \mathrm{~ms}$ expansion of the gas after release from the magnetic trap, a Stern-Gerlach magnetic field with an 80 gauss/cm gradient is applied to spatially separate atoms in the two spin-states [10]. Each component is then resolved separately in absorption images [11] (see inset in Fig. 1).

The emergence of quantum degeneracy is observed through measurements of the equilibrium thermodynamic properties [1] of the two-component gas. In the quantum degenerate regime $\left(T / T_{F} \lesssim 1\right)$, the average energy per particle $E$ rises above the classical expectation $3 k_{B} T$. For the roughly equal $\left(46 \% m_{f}=9 / 2\right)$ mixture of spin states used for evaporation, Fig. 1(a) shows $E$ vs $T / T_{F}$ for each component. The excess energy characteristic of quantum degenerate Fermi systems can clearly be observed in both components. For this data, $T$ and $E$ are determined independently for each component from fits to absorption images of the expanded gas. A fit to the Thomas-Fermi shape expected for an ideal Fermi gas [12] is used to measure $T$, while $E$ is determined from a gaussian fit that is weighted to minimize the fit deviation from the second moment of the image [13]. The widths of both fits are adjusted by roughly $6 \%$ to account for distortions introduced by curvature in the Stern-Gerlach field [14]. The measured temperatures of the two components match to within experimental uncertainty, as expected for thermal equilibrium.

Thermodynamic data for a different spin mixture, $86 \% m_{f}=9 / 2$, are displayed in Fig 1(b). Here the spin composition is controlled by removing some $m_{f}=7 / 2$ atoms after the bulk of the evaporation. Changing the spin mixture manipulates the Fermi energies $E_{F}$ since $E_{F}$ depends on the number of atoms $N$ through $E_{F}=k_{B} T_{F}=\hbar \bar{\omega}(6 N)^{1 / 3}$ [12], where $\bar{\omega}=\left(\omega_{r}^{2} \omega_{z}\right)^{1 / 3}$ is the geometric mean of the harmonic trap frequencies [15]. In the thermodynamics for this less balanced spin mixture, again both components reach quantum degeneracy.

For both mixtures, the thermodynamic data agree well with the ideal gas theory prediction; this indicates that the mean-field energy due to inter-particle interactions in the gas must be small compared to the kinetic and potential energy. For Bose-Einstein condensates (BEC) with similar number, temperature, and scattering lengths, the mean field is quite 
significant. However, the influence of the mean field for a Fermi gas is drastically reduced because the Fermi gas always has higher energy and lower density than a Bose-Einstein condensate. In fact, for our range of experimental conditions $E_{\text {int }} / k_{B} T_{F}<0.4 \%$, where $E_{\text {int }}$ is the interaction (mean field) energy per particle [16].

Also apparent in Fig. 1 is a misalignment of the corresponding $m_{f}=9 / 2$ and $m_{f}=7 / 2$ points on the $T / T_{F}$ axis, indicating that the two components are not equally degenerate. This is particularly true for the $86 \% m_{f}=9 / 2$ case where $E_{F}$ is roughly twice as high for the $m_{f}=9 / 2$ component, and therefore the $m_{f}=9 / 2$ component is always more degenerate. Figure 2 displays the effect of unequal $E_{F}$ by plotting the energy ratio $E_{9 / 2} / E_{7 / 2}$ vs $T / T_{F, 9 / 2}$ ( $T / T_{F}$ for the $m_{f}=9 / 2$ component). For the gas with $86 \% m_{f}=9 / 2$ atoms, $E_{9 / 2} / E_{7 / 2}$ is measured as high as 1.4 in the quantum degenerate regime, strongly violating the classical expectation $E_{9 / 2} / E_{7 / 2}=1$. However, when the gas has roughly equal numbers of $m_{f}=9 / 2$ and $m_{f}=7 / 2$ atoms $E_{F}$ is matched to within $13 \%$, and $E$ is roughly equal for both components irrespective of $T / T_{F}$.

The observed imbalance in $E$ must arise from a change in the collisional interactions in the gas since s-wave collisions would normally redistribute energy equally between the two components. Collisions are predicted to be suppressed by Pauli blocking, a phenomenon common to all Fermi systems such as semiconductors, liquid ${ }^{3} \mathrm{He}$, and nuclear matter. At low $T / T_{F}$, the lowest energy states of the trap are highly occupied and any collision resulting in a final atom state at low energy is suppressed by the Pauli exclusion principle. The energy imbalance is then maintained because collisions that remove energy from the more degenerate component are the most strongly suppressed.

We have directly observed Pauli blocking of elastic collisions in measurements of the thermal equilibration time. The gas is taken out of thermal equilibrium by a rapid removal of high energy atoms from the $m_{f}=7 / 2$ component. Because of gravitational sag in the trap, energy is preferentially removed from the radial direction. As a result, the $m_{f}=7 / 2$ component is initially both out of cross-dimensional equilibrium as well as out of equilibrium with the $m_{f}=9 / 2$ component. A sample data set showing the rethermalization of the energy 
of the $m_{f}=7 / 2$ component is shown in Fig. 3. The cross-dimensional relaxation rate can be used to obtain the elastic collision cross-section [9, 177. One can define an effective collision cross-section $\sigma_{\text {eff }}$ that encapsulates the total effect of Pauli blocking on collisions independent of changes in the density and temperature of the gas. The value of $\sigma_{\text {eff }}$ is predicted to vary from 0 at $T=0$ to the s-wave cross-section $\sigma=4 \pi a^{2}$ in the classical regime [18, where $a$ is the s-wave triplet scattering length.

Figure 4 shows the measured $\sigma_{\text {eff }}$ vs $T / T_{F, 9 / 2}$ for an $86 \% m_{f}=9 / 2$ gas. The rethermalization time constant $\tau$ is extracted from a fit to the time dependence of the $m_{f}=7 / 2$ component aspect ratio, assuming that the energy difference $\delta=E_{x}-E_{z}$ relaxes exponentially $\left(E_{z}\right.$ and $E_{x}$ refer to the $m_{f}=7 / 2$ energy in the axial and one of the radial directions, respectively). The cross-section is then determined through $\frac{1}{\tau}=\frac{n \sigma_{e f f} v}{\alpha}$, where $n=\frac{1}{N_{7 / 2}} \int d^{3} \boldsymbol{r} n_{9 / 2}(\boldsymbol{r}) n_{7 / 2}(\boldsymbol{r})$ is the overlap integral of the density distributions, $v$ is the mean relative speed for a collision between $m_{f}=9 / 2$ and $m_{f}=7 / 2$ atoms, and $\alpha$ is the average number of collisions per $m_{f}=7 / 2$ atom required for cross-dimensional equilibration. The product $n v$ is determined from gaussian fits to the expanded images of each component [19].

For our data, the time dependence of the aspect ratio is complicated by energy transfer from the $m_{f}=9 / 2$ component to the $m_{f}=7 / 2$ component. This is included in the fit to the aspect ratio by assuming that the difference $\Delta=E_{9 / 2}-\eta E_{7 / 2}$ also relaxes exponentially with time constant $\left(\frac{n \sigma_{\text {eff }} v}{A} \frac{N_{7 / 2}+\eta N_{9 / 2}}{N_{9 / 2}(1+\eta)}\right)^{-1}$, where $\eta$ is the equilibrium ratio $E_{9 / 2} / E_{7 / 2}$, and $A$ is a constant similar to $\alpha$. We have used a classical kinetic theory calculation [20] to determine that $\alpha=A=0.75$.

In the classical regime, the measured value of $\sigma_{\text {eff }}$ agrees, within the factor of two systematic uncertainty in atom number, with the best known value of the scattering length for ${ }^{40} \mathrm{~K} a=169 a_{0}$ [21], where $a_{0}$ is the Bohr radius. The effective cross-section drops by a factor of two at $T / T_{F, 9 / 2}=0.2$. Within our uncertainty, the observed size of the Pauli blocking effect agrees with the theoretical value of $\sigma_{\text {eff }}$ from a quantum kinetic calculation [22] shown in Fig. 4. In the calculation, the reduction in $\sigma_{\text {eff }}$ represents the effect of Pauli blocking averaged over all possible initial and final colliding atom states. 
In conclusion, we have cooled an interacting Fermi gas of atoms to one-fifth of the Fermi temperature. At these low temperatures, we have observed strong effects of the quantum statistics on the thermodynamics and collisional dynamics of the gas. Pauli blocking is predicted to cause other modifications in the behavior of the gas including changes in the frequency and damping rate of shape oscillations [16,23],24] and the dipole "scissors-mode" [25] as well as suppression of light scattering [4,26]. In addition, future investigation into the possibility of a paired state BCS-type phase in the atomic Fermi gas will almost certainly require a two-component degenerate system. Realizing this new phase will also require control over the sign and strength of the interactions, which may be possible in ${ }^{40} \mathrm{~K}$ via a predicted Feshbach resonance [27].

This work is supported by the National Institute of Standards and Technology, the National Science Foundation, and the Office of Naval Research. The authors would like to express their appreciation for useful discussions with C.E. Wieman, E.A. Cornell, and M. Holland. 


\section{REFERENCES}

* Quantum Physics Division, National Institute of Standards and Technology

[1] B. DeMarco and D.S. Jin, Science 285, 1703 (1999).

[2] K. Gibble and B. Verhaar, Phys. Rev. A 52, 3370 (1995).

[3] S.-K. Yip and T.-L. Ho, Phys. Rev. A 59, 4653 (1999).

[4] B. DeMarco and D.S. Jin, Phys. Rev. A 58, R4267 (1998).

[5] J.M.K.V.A. Koelman et al., Phys. Rev. Lett. 59, 676 (1987); G. Ferrari, Phys. Rev. A 59, R4125 (1999).

[6] M. Houbiers et al., Phys. Rev. Lett. 56, 4864 (1997); T.-L. Ho and V.B. Shenoy, J. Low Temp. Phys. 111, 937 (1998).

[7] H.T.C. Stoof, M. Houbiers, C.A. Sackett, and R.G. Hulet, Phys. Rev. Lett. 76, 10 (1996); M.J. Bijlsma, B.A. Heringa, and H.T.C. Stoof, Phys. Rev. A 61, 053601 (2000).

[8] S. Inouye et al., Nature (London) 392, 151 (1998); Ph. Courteille et al., Phys. Rev. Lett. 81, 69 (1998); J.L. Roberts et al., Phys. Rev. Lett. 81, 5109 (1998).

[9] B. DeMarco, J.L. Bohn, J.P. Burke, M. Holland, and D.S. Jin, Phys. Rev. Lett. 82, 4208 (1999).

[10] D.M. Stamper-Kurn et al., Phys. Rev. Lett. 80, 2027 (1998).

[11] A controlled mixture of laser polarization is used for resonant absorption imaging in order to avoid lensing caused by the high density gradients present in the gas.

[12] D.A. Butts and D.S. Rokhsar, Phys. Rev. A 55, 4346 (1997).

[13] We have checked that the weighted fit returns the correct value of $E$ for calculated FermiDirac, Bose-Einstein, and classical distributions. We measure the expansion energy per particle, and plot $E$ (twice the expansion energy) assuming that mean field effects are 
small [23].

[14] The correction factor is measured across the range of temperatures used in this experiment and is consistent with a classical calculation describing the individual trajectories of atoms experienced during the expansion.

[15] The two spin-states have different magnetic moments and therefore experience slightly different harmonic oscillator frequencies. The magnetic trap frequencies for all of the experiments described in this Letter are $134.0 \mathrm{~Hz}$ radial and $19.9 \mathrm{~Hz}$ axial for the $m_{f}=9 / 2$ atoms. The corresponding frequencies for the $m_{f}=7 / 2$ atoms are reduced by the square root of the ratio of the magnetic moments $(\sqrt{7 / 9})$.

[16] M. Amoruso et al., Eur. Phys. J. D 8, 361 (2000).

[17] C.R. Monroe et al., Phys. Rev. Lett. 77, 414 (1993).

[18] See, for example, a discussion of Galitskii's work in A.L. Fetter and J.D. Walecka, Quantum Theory of Many-Particle Systems (McGraw-Hill, San Francisco, 1971).

[19] Using gaussian fits as opposed to Fermi-Dirac distributions makes at most a $2 \%$ error in the product $n v$ for $T / T_{F}>0.2$.

[20] We solve Enskog's equation to determine the time dependence of $\Delta$ and $\delta$. See for example F. Reif, Fundamentals of Statistical and Thermal Physics, (McGraw-Hill ,1965).

[21] H. Wang et al., Phys. Rev. A 62, 052704 (2000).

[22] M.J. Holland, B. DeMarco, and D.S. Jin, Phys. Rev. A 61, 053610 (2000).

[23] G.M. Bruun and C.W. Clark, Phys. Rev. A 61, 061601 (R) (2000).

[24] L. Vichi and S. Stringari, Phys. Rev. A 60, 4734 (1999).

[25] A. Minguzzi and M.P. Tosi, Phys. Rev. A 63, 023609 (2001).

[26] K. Helmerson, M. Xiao, and D. Pritchard, International Quantum Electronics Confer- 
ence 1990, book of abstracts (IEEE, New York, 1990), abstr. QTHH4; Th. Busch, J. R. Anglin, J.I. Cirac, P. Zoller, Europhys. Lett. 44, 1 (1998); J. Ruostekoski and J. Javanainen, Phys. Rev. Lett. 82, 4741 (1999).

[27] J.L. Bohn, Phys. Rev. A 61, 053409 (2000). 
FIGURES

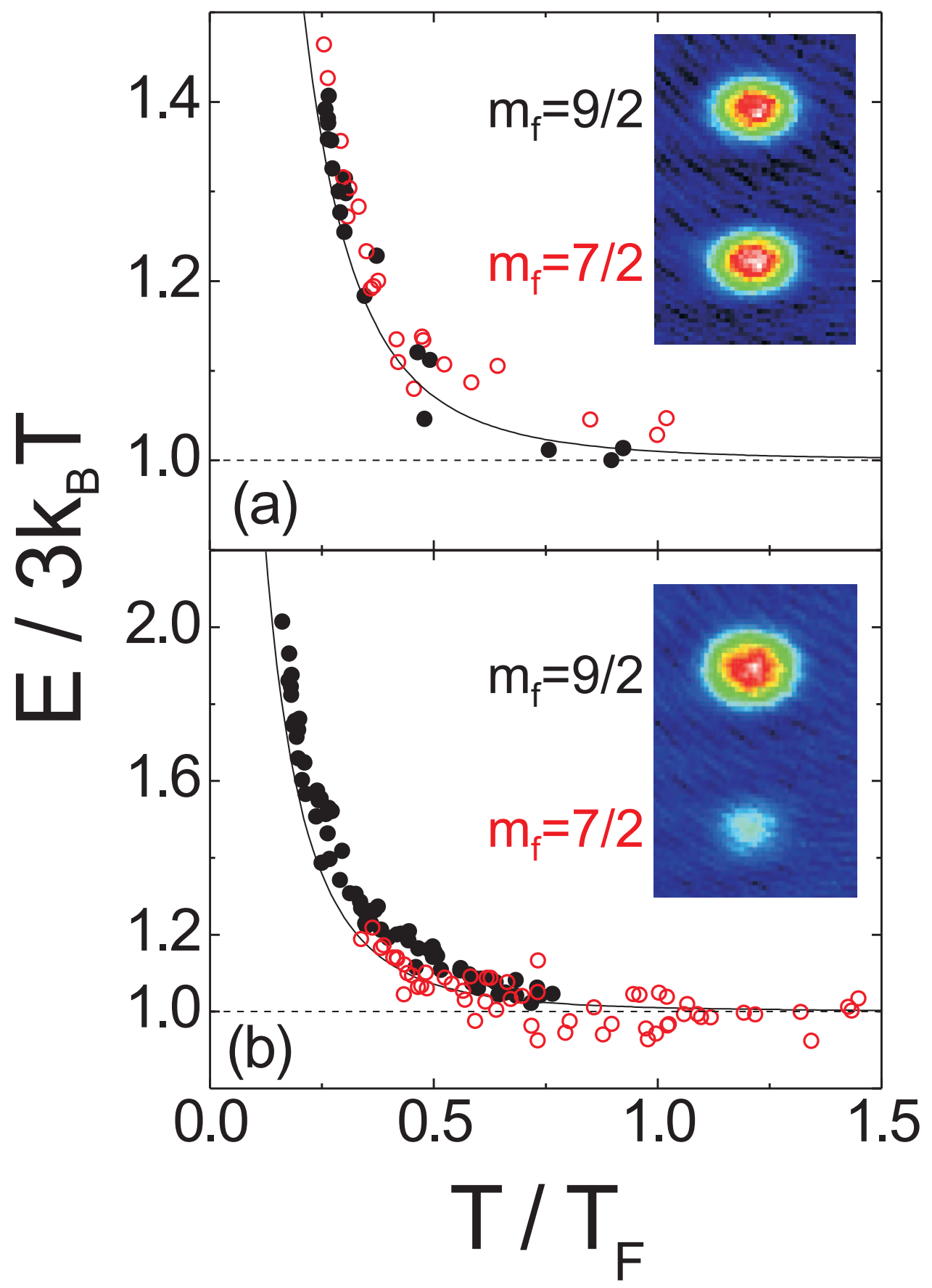


FIG. 1. Thermodynamics of the interacting gas. The average energy per particle $E$, extracted from absorption images such as the examples shown in the insets, is displayed for two spin mixtures, $46 \% m_{f}=9 / 2(\mathrm{a})$ and $86 \% m_{f}=9 / 2(\mathrm{~b})$. In the quantum degenerate regime, the data deviate from the classical expectation (dashed line) as the atoms form a Fermi sea arrangement in the energy levels of the harmonic trapping potential. The data in (a) represent the spin mixture used for evaporation, where we reach $T / T_{F} \sim 0.25$ at $90 \mathrm{nK}$ and $N=2.8 \times 10^{5}$ atoms. The data agree with the ideal Fermi gas prediction for a harmonic trap, shown by the solid line. Misalignment of corresponding $m_{f}=9 / 2$ and $m_{f}=7 / 2$ points on the $T / T_{F}$ axis reflects a difference in the Fermi energies for the two components.

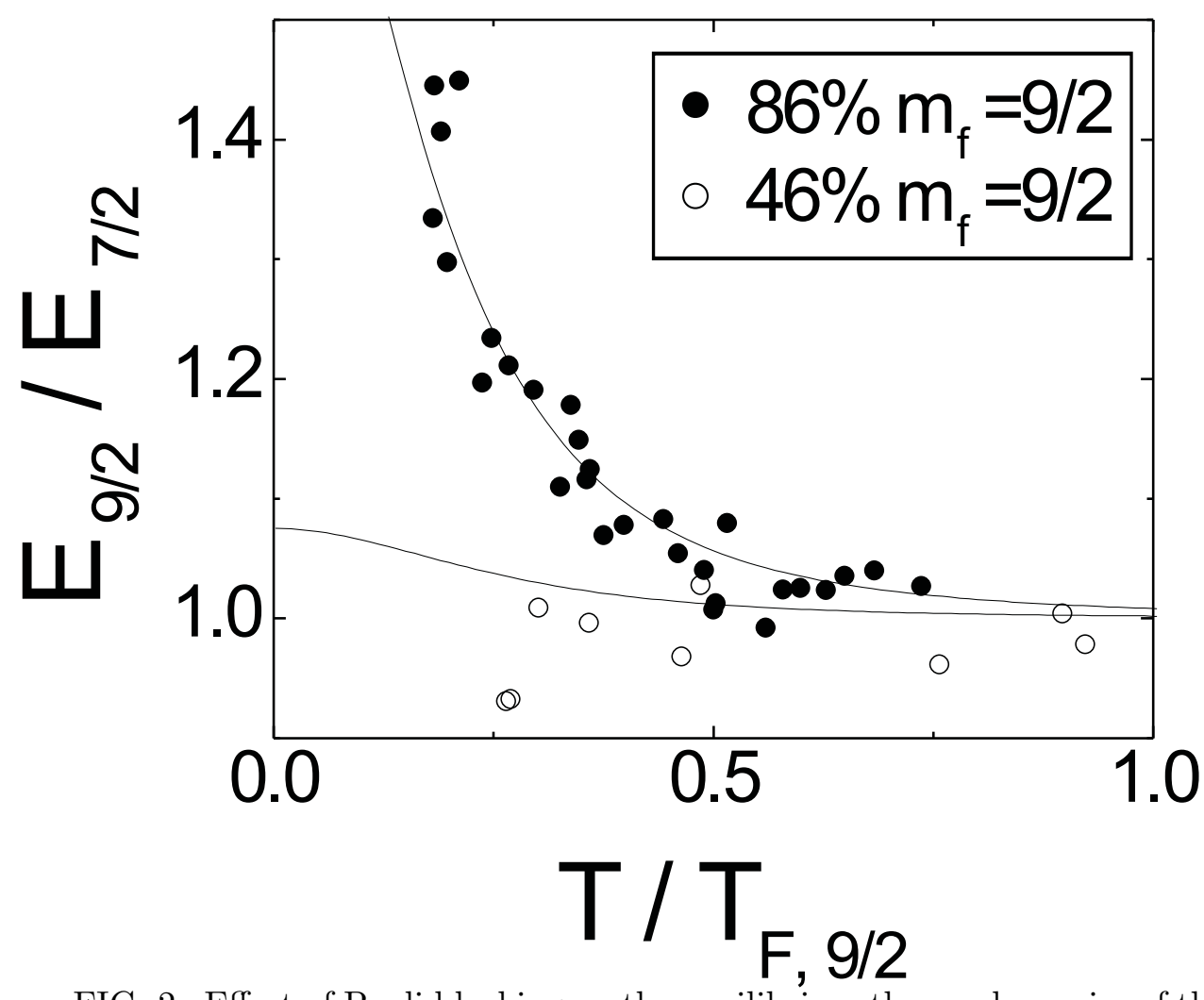

FIG. 2. Effect of Pauli blocking on the equilibrium thermodynamics of the gas. Using the same data shown in Fig. 1, the ratio of energy, $E_{9 / 2} / E_{7 / 2}$, for pairs of clouds from each double image is plotted vs. $T / T_{F, 9 / 2}$. Each point in this plot represents the average of two runs of the experiment. For comparison, the prediction for an ideal Fermi gas is shown by the solid lines. The energy imbalance revealed at low $T / T_{F}$ is maintained by Pauli blocking of collisions. 


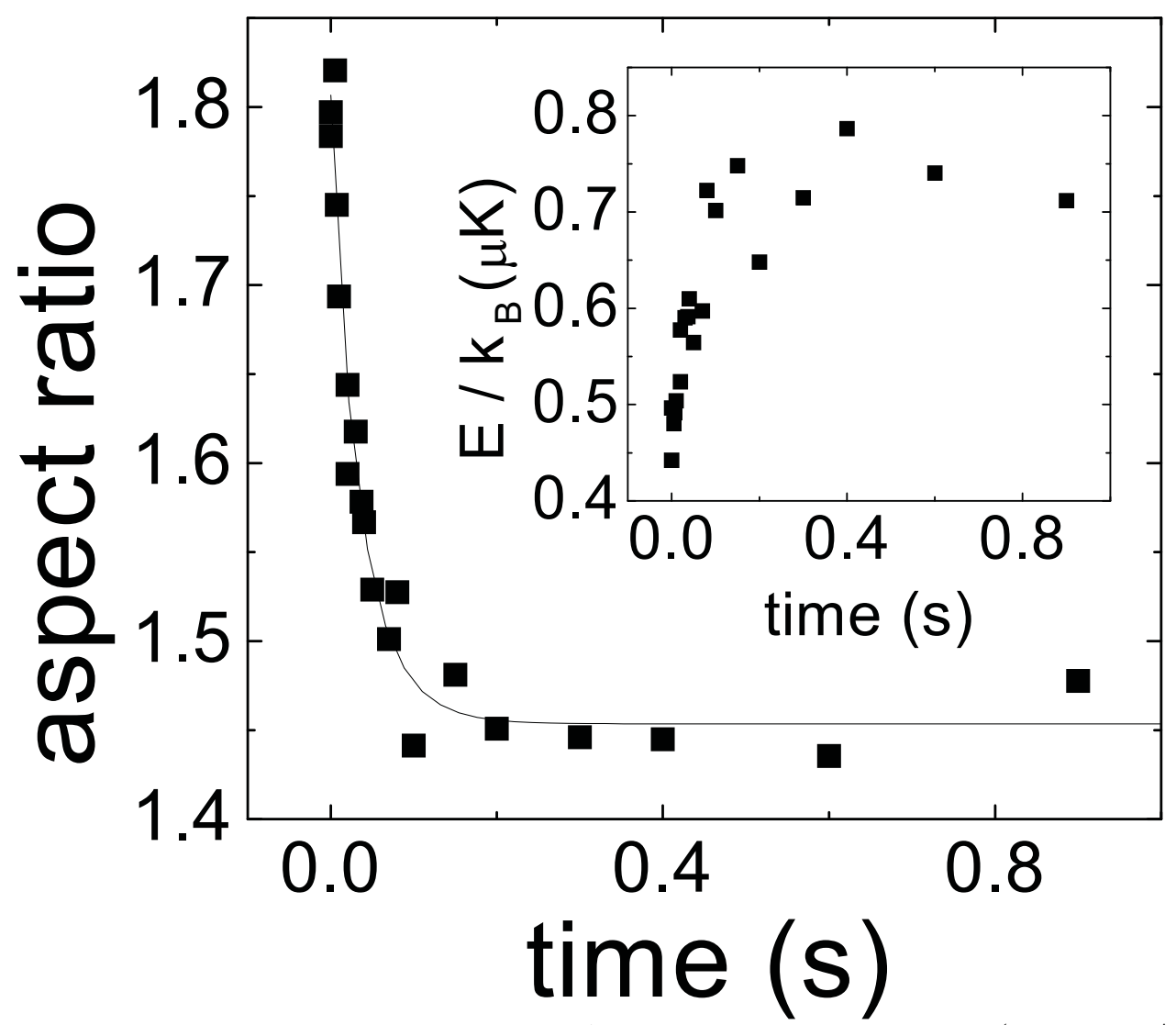

FIG. 3. Rethermalization data. A plot of the aspect ratio (axial size/radial size) of the expanded $m_{f}=7 / 2$ component vs. time shows cross-dimensional energy rethermalization. The inset, a plot of the average energy per particle of the $m_{f}=7 / 2$ component, shows the simultaneous transfer of energy from the $m_{f}=9 / 2$ to $m_{f}=7 / 2$ component. The data in this figure were taken for a gas with $T / T_{F, 9 / 2}=0.5, N_{9 / 2}=4.6 \times 10^{5}$, and $N_{7 / 2}=7.7 \times 10^{4}$. A fit (solid lines) of the time dependent aspect ratio is used to measure the effective collision cross-section. 


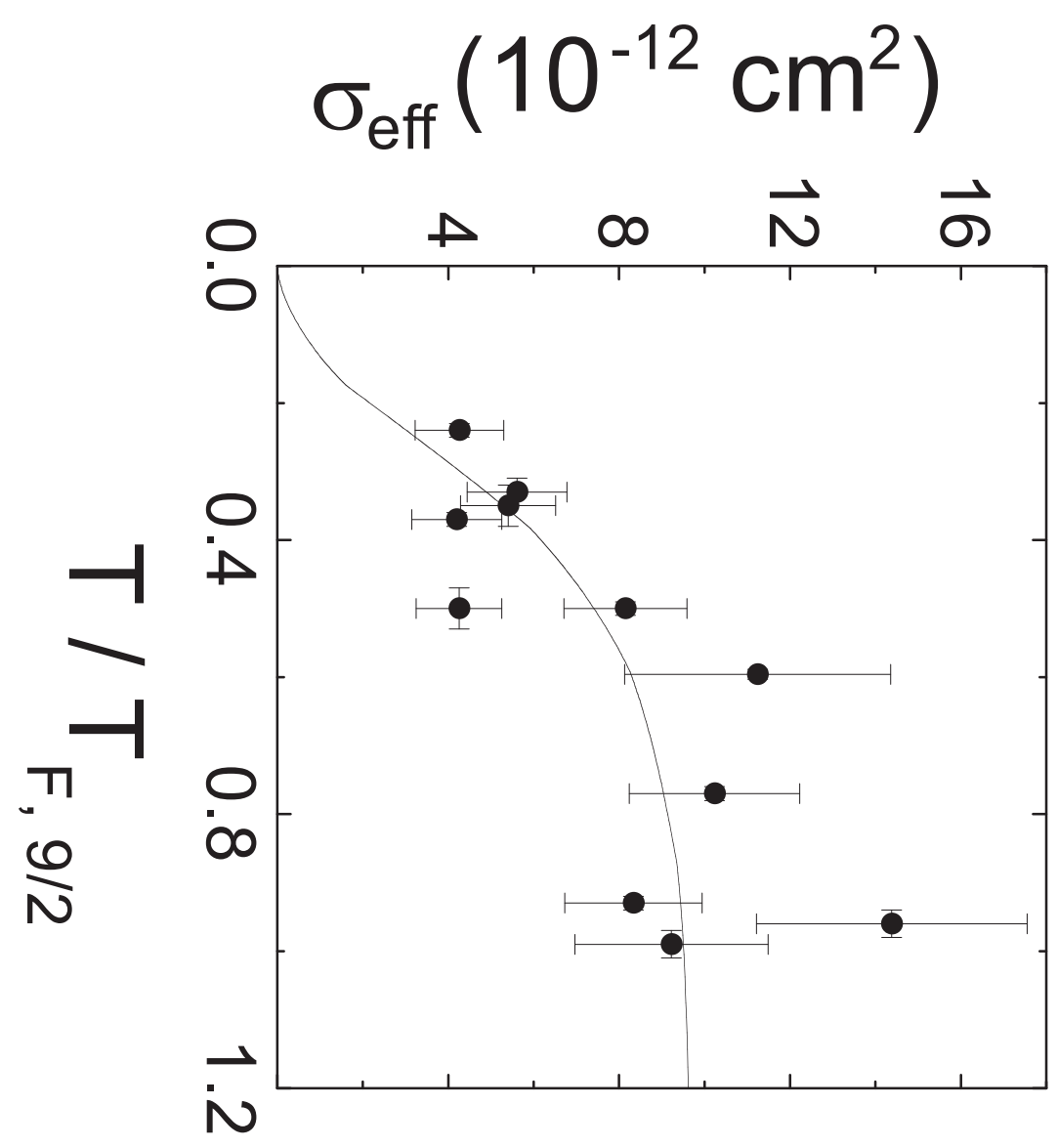


FIG. 4. Collisional Pauli blocking. A factor of two decrease in the effective elastic collision cross-section, $\sigma_{\text {eff }}$, is observed at low $T / T_{F}$. The error bars in $\sigma_{\text {eff }}$ are predominately from uncertainty in the fits to the time dependence of the $m_{f}=7 / 2$ aspect ratio, while scatter in number and temperature set the error bars in $T / T_{F}, 9 / 2$. In addition, there is a $26 \%$ systematic uncertainty in $T / T_{F, 9 / 2}$ and a factor of two systematic uncertainty in $\sigma_{\text {eff }}$ from uncertainty in the number determined from absorption imaging. The solid line shows the result from a quantum kinetic calculation of the collision rate. At high $T / T_{F}$ the data agree with the known value of the s-wave collision cross-section, and at low $T / T_{F}$ the observed decrease in $\sigma_{\text {eff }}$ agrees with the quantum kinetic prediction. 